\title{
Adicción a redes sociales, Miedo a perderse experiencias (FOMO) y Vulnerabilidad en línea en estudiantes universitarios
}

\author{
Manuel Varchetta \\ Dipartimento di Psicologia, “Sapienza” Università di Roma, Roma, Italia ${ }^{1}$ \\ https://orcid.org/0000-0001-8111-6715 \\ Angelo Fraschetti ${ }^{1}$ \\ https://orcid.org/0000-0003-1701-5789 \\ Emanuela Mari ${ }^{1}$ \\ https://orcid.org/0000-0003-2367-3139 \\ Anna Maria Giannini ${ }^{1}$ \\ https://orcid.org/0000-0002-0614-4457
}

Recibido:22/05/2020Ｒevisado: 12/06/2020Ｐublicado:22/06/2020

\begin{abstract}
Resumen
Si bien existe un crecimiento en investigación sobre las redes sociales, es necesario señalar que las asociaciones entre el abuso de estas plataformas, el fenómeno del Fear of Missing Out (FOMO) o Miedo a perderse experiencias y de la Vulnerabilidad en línea han sido poco estudiadas. En ese sentido, esta investigación tiene como objetivo proporcionar un marco de las actitudes y comportamientos relacionados con el uso de las redes sociales en jóvenes universitarios. Para ello, se realizó una muestra que contó con la participación de 306 estudiantes universitarios de 18 a 30 años (152 mujeres y 154 hombres; edad promedio = 21.8; desviación estándar = 3.19) y respondió a un cuestionario en línea, respecto al uso de las redes ociales, Fear of Missing Out (FOMO), Necesidades psicológicas básicas, Auto-estima y Vulnerabilidad en línea. El análisis de correlaciones mostró una relación positiva entre el FOMO, la Vulnerabilidad en línea y la Adicción a redes sociales. Los resultados evidenciaron que las mujeres tienen mayores niveles de adicción a redes sociales y de necesidad de apoyo. El análisis de regresión mostró que el FOMO es el mejor predictor de la Adicción a redes sociales. Finalmente, se discuten las repercusiones en el ámbito educativo de los resultados.
\end{abstract}

Palabras clave: Adicción a redes sociales; FOMO; Vulnerabilidad en línea; Motivación humana; Estudiantes universitarios.

\section{Social Media Addiction, Fear of Missing Out (FoMO) and Online Vulnerability in university students}

\footnotetext{
Abstract

Despite the growing research on social networks sites (SNS), the associations between the abuse of these platforms, the phenomenon of Fear of Missing Out (FOMO) and Online Vulnerability has been understudied. The aim of this research was to provide a framework of attitudes and behaviors related to the use of the social network in young university students.
} 
In this study, the sample was composed to 306 Italian university students aged 18 to 30 (152 women; mean of age = 21.8; standard deviation = 3.19) who responded to an online survey regarding their SNS behaviors, Fear of Missing Out (FOMO), Basic Psychological Needs, Self-Esteem and Online Vulnerability. Correlations analysis showed a positive relationship between FOMO, Online Vulnerability, and Social Media Addiction. The results showed that women have a higher level of Social Media Addiction and the Need for Relatedness. The regression analysis showed that FOMO is the best predictor of Social Media Addiction. Implications of the findings in the educational field are discussed.

Keywords: Social Media Addiction; FOMO; Online Vulnerability; Human motivation; University students.

\title{
Dependência de Redes Sociais, Fear of Missing Out (FoMO) e Vulnerabilidade Online em estudantes universitários
}

\begin{abstract}
Resumo
Apesar da crescente pesquisa sobre as redes sociais, as associações entre o abuso destas plataformas, o fenômeno do Fear of Missing Out (FOMO) e da Vulnerabilidade Online foram pouco estudadas. Esta investigação destina-se a proporcionar um quadro de atitudes e comportamentos relacionados com a utilização das redes sociais em jovens universitários. Neste estudo, a amostra foi composta por 306 universitários de 18 a 30 anos (152 mulheres; idade média = 21,8; desvio padrão = 3.19) e respondeu a um questionário on-line sobre o uso das redes sociais, Fear of Missing Out (FOMO), Necessidades psicológicas básicas, Auto-estima e Vulnerabilidade online. A análise de correlação mostrou uma relação positiva entre o FOMO, a Vulnerabilidade online e a dependencia de redes sociais. Os resultados mostraram que as mulheres têm um nível mais alto de dependência de redes sociais e de necessidade de apoio. A análise de regressão mostrou que o FOMO é o melhor preditor da Dependencia de redes sociais. Finalmente, discutem-se as implicações em âmbito educativo dos resultados.

Palavras-chave: Dependência de redes sociais; FOMO; Vulnerabilidade Online; Motivação Humana; Estudantes
\end{abstract} universitarios.

Como citar:

Varchetta, M., Fraschetti, A., Mari, E., y Giannini, A. M. (2020). Adicción a redes sociales, Miedo a perderse experiencias (FOMO) y Vulnerabilidad en línea en estudiantes universitarios. Revista Digital de Investigación en Docencia Universitaria, 14(1), e1187. https://doi.org/10.19083/ridu.2020.1187

E 1 desarrollo de la World Wide Web ha cambiado por completo la forma en que las personas se comunican entre sí y difunden información (Krämer \& Winter, 2008). Entre los sitios más exitosos en la escena virtual se encuentran las redes sociales. Consideradas una fuerza dominante en la sociedad actual basada en la digitalización, ofrecen la oportunidad de desarrollar y mantener su esfera social en entornos interactivos y ricos en contenido multimedia en línea (Boyd \& Ellison, 2007). El uso de las redes sociales puede proporcionar una amplia gama de oportunidades para sus usuarios: promueve el capital social, lo que a su vez fortalece las conexiones con las personas y las redes, puede mejorar la comunicación entre miembros de la familia y amigos cercanos (Li \& Chen, 2014), pueden usarse para mantener relaciones y conectarse (Joinson, 2008), están asociados con la conexión, un mayor apoyo social y una reducción de la soledad (Burke, Marlow \& Lento, 2010; Kim \& Lee, 2011; Kim \& Tussyadiah, 2013). Además, el uso de las redes sociales se considera una de las herramientas disponibles para promover la ciudadanía global entre los jóvenes (UNESCO, 2014).

También es importante señalar que el uso de algunas aplicaciones puede estar motivado por diferentes razones. Por ejemplo, Villacampa, Ingram, Martí-Vilar \& Oliver-La Rosa (2018), han observado que Facebook puede ser una herramienta que fa- 
vorece un mayor interés para las actividades prosociales respecto a las sexuales y eso no diferiría según el sexo de los participantes. En cambio, una investigación emergente sobre Tinder, una aplicación de citas móviles, sugiere que los usuarios ven esta aplicación como una herramienta útil para la búsqueda de sexo ocasional (Lefebvre, 2017; Sumter, Vandenbosch \& Ligtenberg, 2017). Es posible comprobar que Tinder puede ser utilizado para buscar actividades sexuales fuera del contexto de una relación romántica, (Weiser, Niehuis, Flora, Punyanunt-Carter, Arias, \& Baird, 2017).

Por otro lado, la dinámica que experimentó el escenario tecnológico provocó una serie de deficiencias en el mundo real, y con ello, la búsqueda continua de experiencias virtuales. De hecho, la percepción de la necesidad de estar en línea puede conducir al uso compulsivo de las redes sociales, que en casos extremos puede causar síntomas y consecuencias tradicionalmente asociadas al abuso de sustancias (Kuss \& Griffiths, 2011; Andreassen, 2015). Ello pondría en riesgo las relaciones familiares y sociales, además de la salud psicofísica.

Recientemente, ha crecido la atención por un nuevo fenómeno social, el Fear of Missing Out (FOMO) o Miedo a perderse experiencias. Este término se refiere a la sensación de inquietud, a menudo intensa, desencadenada por la preocupación de que amigos $u$ otros puedan presenciar experiencias gratificantes particulares de las cuales se está ausentes (Przybylski, Murayama, DeHann \& Gladwell, 2013). Es el miedo a quedarse fuera, pensar constantemente que otros están haciendo algo más interesante y gratificante que nosotros y que nos estamos perdiendo algo. Se caracteriza por el deseo de permanecer socialmente conectado y podría manifestarse como una forma de ansiedad social (Przybylski et al., 2013).

La teoría de la autodeterminación (Self-Determination Theory, SDT) de Deci \& Ryan (1985), una macro-teoría de la motivación humana, proporciona una perspectiva útil para enmarcar una comprensión empírica del FOMO. Según la SDT, la autorregulación y la salud psicológica se basan en la satisfacción de tres necesidades psicológicas básicas: Competencia, o sea la capacidad de actuar eficazmente en el mundo; Autonomía, es decir autoría o iniciativa personal; Apoyo, es decir cercanía o conexión con los demás. Investigaciones realiza- das en contextos educativos (Deci \& Ryan, 2000) y de videojuegos (Przybylski, Weinstein, Ryan \& Rigby, 2009) indican que la satisfacción de estas necesidades básicas está fuertemente asociada con la regulación del comportamiento. A través de este marco teórico, el fenómeno FOMO puede entenderse como una especie de "limbo auto-regulatorio" (Przybylski et al., 2013), resultante de déficits situacionales o crónicos en las satisfacciones psicológicas de las necesidades básicas. Además, se ha sugerido que las personas con niveles más altos de FOMO pueden entrar en un ciclo de comportamientos que van a la búsqueda de una reafirmación de su propia identidad y autoestima pasando una mayor cantidad de tiempo en línea. Ello, a su vez, puede conducir a un mayor temor a perder experiencias gratificantes, una mayor capacidad para comportamientos reveladores y amistosos y, en última instancia, una mayor disminución del bienestar social y psicológico (Przybylski et al., 2013).

La preferencia por la comunicación en línea, caracterizada por la ausencia de lenguaje no verbal, expresiones faciales, gestos, etc. podría aumentar el riesgo, especialmente para los jóvenes, de no alcanzar una madurez emocional adecuada. Goleman (1995), recuerda cómo la base de la alfabetización emocional es la empatía, es decir, la capacidad de reconocer emociones y sentimientos en los demás. Idealmente, poniéndose en el lugar de los demás y logrando conectar comportamientos y estados emocionales, el sujeto aprende a reconocer y dar sentido a sus emociones. Por el contrario, la incapacidad de reconocer las emociones del otro, impide la comprensión de las propias, llevando al desinterés emocional y, en el peor de los casos, al comportamiento antisocial. El intercambio de imágenes, videos y comentarios detrás de una pantalla, en ausencia total de contacto humano directo, puede hacer interacciones caracterizadas por mecanismos de falta de empatía, base de algunos mecanismos de desconexión moral y deshumanización hacia el interlocutor (Sgalla \& Giannini, 2018).

El uso excesivo de la red en prácticas que incluyen la autopresentación y la participación a grandes redes en línea puede hacer que los usuarios sean más vulnerables a experimentar formas de Ciberbullying como las mencionadas anterior- 
mente (Buglass, Binder, Betts \& Underwood, 2016; Staksrud, Ólafsson, \& Livingstone, 2013). A este respecto, hablamos de Vulnerabilidad en línea, definida como la tendencia de un individuo a experimentar daños que socavan su bienestar psico-físico y su reputación (Davidson \& Martellozzo, 2012) como resultado de participación en algunas actividades en línea. La exposición a la Vulnerabilidad en línea no implica automáticamente una vulnerabilidad psicológica básica (Livingstone \& Smith, 2014). Sin embargo, existe evidencia convincente que sugiere que estar expuesto a episodios de Vulnerabilidad en línea puede tener consecuencias negativas para el bienestar psicológico de un usuario (Patchin \& Hinduja, 2010; Keipi, Oksanen, Hawdon, Näsi \& Räsänen, 2015). Los aumentos recientes y sustanciales en la prevalencia de tales experiencias negativas en línea (Jones, Mitchell \& Finkelhor, 2013) se han relacionado con consecuencias perjudiciales como la depresión (Landoll, La Greca, Lai, Chan \& Herge, 2015) hasta llegar al suicidio (Patchin \& Hinduja, 2010, Washington Post, 2013).

Esta investigación tiene como objetivo proporcionar un marco de las actitudes y comportamientos relacionados al uso de las redes sociales de jóvenes universitarios italianos; estudiar las variables psicológicas que pueden relacionarse con tal uso y entre sí e investigar sobre su posibles diferencias según el género. Además, se quiere comprobar en qué medida el FOMO, aplicado al uso de las redes sociales durante las principales actividades diarias, puede predecir la aparición de síntomas adictivos asociados con el uso de estas.

\section{Método}

\section{Diseño}

Esta investigación contó con un diseño transversal de tipo descriptivo, puesto que se profundizaron las modalidades, características y frecuencias del uso de la red, se investigaron eventuales diferencias de género en las variables estudiadas. Asimismo, es correlacional, pues se explora la relación entre el uso de redes sociales, FOMO, necesidades psicológicas básicas, comportamientos de autopromoción y Vulnerabilidad en línea.

\section{Participantes}

La muestra fue no probabilística y por conveniencia. Los participantes fueron seleccionados mediante dos criterios: ser estudiantes universitarios y tener una edad comprendida entre los 18 y 30 años. Participaron a la investigación 306 estudiantes universitarios de la Universidad "La Sapienza" de Roma, Italia. La muestra estuvo compuesta por 152 mujeres (49.7\%) y 154 hombres (50.3\%), edad promedio de 21.80 ( $D E=3.19$ ). Casi todos los participantes se declararon solteros (96.1\%). No se consideraron eventuales diferencias en cuanto a la facultad de pertenencia de cada estudiante.

En Italia los programas de grado comprenden tres años de estudio. Aquellos de master o postgrado prevén dos años de cursos (primer ciclo universitario: $70.3 \%$; máster o postgrado: $29.7 \%$ ).

\section{Instrumentos}

La encuesta en línea constó de tres secciones. En la primera sección, se solicitó información socio-demográfica: edad, género, estado civil y año de carrera universitaria (grado; master/postgrado). En la segunda sección se solicitaron informaciones sobre el uso de la tecnología: frecuencia de uso de las redes sociales durante el horario universitario (opciones de respuesta entre $1=$ Nunca y 5 = Siempre); preferencia de uso de aplicaciones de redes sociales (Facebook, Instagram, WhatsApp, etc.). Tiempo de utilización de las redes sociales (diferentes opciones de respuesta entre 1 = no lo uso y 5 = más de 5 horas).

En la tercera sección se utilizó una batería de escalas prestablecidas:

\section{Social Media Engagement Scale, SMES (Przybyls- ki, Murayama, DeHann \& Gladwell, 2013).}

La escala presenta 5 ítems con el objetivo de cuantificar la frecuencia de uso de las redes sociales durante las principales actividades diarias. Los participantes utilizaron una escala de estilo Likert de ocho puntos de 1 (Ningún un día la semana pasada) a 8 (Todos los días la semana pasada). Los análisis de componentes principales indicaron los cinco ítems cargados en un solo factor, explicando el 59.27\% de la variabilidad observada (Przybylski et al., 2013), por lo que se sumaron los puntajes para crear una puntación media para cada participante $(\mathrm{a}=.82)$. 
Bergen Social Media Addiction Scale, BSMAS (Andreassen, Billieux, Griffiths, Kuss, Demetrovics, Mazzoni \& Pallesen, 2016).

Es una adaptación de la Bergen Facebook Addiction Scale (BFAS; Andreassen, Torsheim, Brunborg y Pallesen, 2012). La confiabilidad y la consistencia interna del BSMAS fue respaldada por varios indicadores (Andreassen et al., 2012; Andreassen, Billieux, et al., 2016). Contiene seis elementos que reflejan elementos básicos de las adicciones (Griffiths, 2005). Los participantes respondieron a los ítems (por ejemplo, ¿utilizó tanto las redes sociales que su uso ha tenido un impacto negativo en sus estudios?; ¿Intentó dejar de usar las redes sociales sin éxito?) usando una escala Likert de 5 pasos, de 1 (Muy raramente) a 5 (Muy a menudo), produciendo una puntuación compuesta de 1 a 5, La traducción italiana de BSMAS resulta en un instrumento psicométricamente robusto, con una buena consistencia interna, con un a de Cronbach igual a .88 (Monacis, De Palo, Griffiths \& Sinatra, 2017).

\section{Fear of Missing Out Scale, FOMOs (Przybylski,} Murayama, DeHann \& Gladwell, 2013).

La escala muestra una buena consistencia interna $(a=.90)$ y validez (Przybylski et al., 2013). El instrumento ofrece 10 ítems en forma de afirmación (por ejemplo, cuando voy de vacaciones, vigilo lo que hacen mis amigos; cuando hago algo divertido, es importante que comparta detalles en línea). Para responder, se utilizó una escala Likert de cinco pasos ( 1 = No es cierto para mí; 5 = Extremadamente cierto para mí). La escala produjo un puntaje promedio de 1 a 5, con puntajes más altos que indicaban mayores niveles de FOMO.

\section{Basic Psychological Needs Scale, BPNS (La Guar-} dia, Ryan, Couchman \& Deci, 2000).

La escala tiene 21 ítems presentados en una escala Likert de 5 puntos de 1 (No es cierto en absoluto) a 5 (Es totalmente cierto). La versión italiana de la escala tiene buenas propiedades psicométricas ( $\mathrm{a}=.82$ ) y una validez convergente con diferentes medidas de comportamiento pro-social, apego seguro y bienestar (Szadejko 2003). La escala toca tres dimensiones: Autonomía: (puedo decidir libremente cómo vivir mi vida); Competencia (a menudo no me siento muy competente); Apoyo: (en general, las personas son lo suficientemen- te amigables conmigo). Calculamos los puntajes para cada participante al calificar de manera inversa los elementos redactados negativamente y luego promediar las respuestas.

Rosenberg Self-Esteem Scale, RSE (Rosenberg, 1965) en la versión italiana de Prezza, Trombaccia \& Armento (1997).

El RSE contiene 10 ítems redactados positivamente (por ejemplo, en general, estoy satisfecho conmigo mismo) y negativo (por ejemplo, a veces creo que no soy bueno en absoluto). Las respuestas se dieron en una escala Likert de 4 puntos que varía de 0 (Totalmente en desacuerdo) a 3 (Totalmente de acuerdo). Los elementos negativos se recodificaron y se calcularon al revés. El RSE se desarrolló originalmente para su uso con muestras de adultos para las cuales ha demostrado una buena consistencia interna $(=.86)$ y validez de constructo (Robins y Trzesniewski, 2001). La escala va de 0 a 30. Las puntuaciones entre 15 y 25 están en el rango de normalidad. Puntuaciones inferiores a 15 indican una baja autoestima.

\section{Online Vulnerability Scale (Buglass, Binder, Betts \& Underwood, 2017).}

La escala incluye 6 ítems que combinaban preguntas y teorías presentadas por Binder, Howes \& Smart (2012). El instrumento investiga con qué frecuencia, al usar las redes sociales, las personas experimentan personalmente o ven a otros encontrarse con situaciones o contenido de naturaleza violenta, embarazosa, dañina o no deseada (por ejemplo, comentarios críticos y / u ofensivos; contenido sexual o violento; stalking o abuso en línea). El método de respuesta se refería a una escala Likert de 5 pasos de 1 (Muy raramente) a 5 (Muy a menudo). La escala mostró buenas propiedades psicométricas con a de Cronbach igual a 91 (Buglass et al., 2017). Las respuestas produjeron un puntaje promedio de 1 a 5, con puntajes más altos que indicaban una mayor exposición a la vulnerabilidad durante el uso de las redes sociales.

\section{Procedimientos}

Los participantes completaron una encuesta en línea en seguridad, optimizada para su uso en 
computadoras, tabletas y dispositivos móviles. Fue utilizada la plataforma Moodle de Google para la difusión del cuestionario. Tras haber leído el consentimiento informado, cada persona pude decidir voluntariamente participar en la investigación, empezando a responder a la encuesta de manera digital. Se les garantizó la máxima confidencialidad en el manejo y en el análisis de las respuestas. Los datos se recopilaron durante un período de 6 meses, de febrero 2019 a julio 2019.

Los tiempos de administración de toda la encuesta fueron de aproximadamente 15 minutos.

\section{Análisis de datos}

Para el análisis estadístico se utilizó el software IBM SPSS versión 25. En una primera fase de análisis, se llevaron a cabo las principales estadísticas descriptivas (frecuencia, media y desviación estándar) con respecto a la información general y las escalas utilizadas. Posteriormente, se realizaron análisis de correlación utilizando el coeficiente $r$ de Pearson. Además, se calcularon las correlaciones de Pearson para los grupos de hombres y de mujeres por separado y las comparamos usando la prueba $z$ de Fisher (Lenhard \& Lenhard, 2014) para verificar la significatividad de las diferencias entre los dos grupos. Se aplicó la prueba $t$ de Student para comprobar la existencia de diferencias de género en las variables analizadas. Se calculó el tamaño del efecto mediante la $d$ de Cohen. Por úl- timo, se hizo un análisis de regresión lineal, con el fin de comprobar si las variables independientes SMES y FOMO fueran capaces de predecir significativamente la dependiente BSMAS.

\section{Resultados}

El 70.9\% de los participantes usa a menudo y siempre las redes sociales durante el horario universitario. Solo el 2\% declara no usarlos. Entre las aplicaciones más utilizadas encontramos WhatsApp (96.4\%), utilizado para más de 3 horas al día del 63\% de la muestra total, Instagram (95.1\%) visitado más de 3 horas al día por el $41.8 \%$ de la muestra y Facebook (69.9\%) usado menos de 1 hora al día. El 77.5\% de los participantes usan las redes sociales "todos los días" en los 15 minutos antes de acostarse y el $55.6 \%$ en los 15 minutos después de despertarse.

Las correlaciones globales entre las variables observadas y las puntaciones medias obtenidas en las escalas se presentan en la Tabla 1. En general, el FOMO está directamente correlacionado, con alta intensidad con la variable BSMAS ( $r=.73$, $p<.01)$. Se observan correlaciones moderadamente positivas entre FOMO y la Vulnerabilidad en línea $(r=.38, p<.01)$.

Además, el BSMAS está positivamente correlacionado, con intensidad moderada, con la Vulnerabilidad en línea $(r=.32, p<.01)$.

Tabla 1

\begin{tabular}{|l|c|c|c|c|c|c|c|c|c|c|}
\hline Variables & $\mathbf{M}$ & $\mathbf{D E}$ & $\mathbf{1}$ & $\mathbf{2}$ & $\mathbf{3}$ & $\mathbf{4}$ & $\mathbf{5}$ & $\mathbf{6}$ & $\mathbf{7}$ & $\mathbf{8}$ \\
\hline 1. SMES & 4.88 & 1.75 & - & & & & & & & \\
\hline 2. BSMAS & 2.21 & 0.81 & $.53^{* *}$ & - & & & & & & \\
\hline 3. FOMO & 2.23 & 0.80 & $.43^{* *}$ & $.73^{* *}$ & - & & & & & \\
\hline 4. BNPS (Autonomía) & 3.74 & 0.64 & $-.15^{* *}$ & $-.49^{* *}$ & $-.50^{* *}$ & - & & & & \\
\hline 5. BNPS (Competencia) & 3.41 & 0.72 & $-.16^{* *}$ & $-.45^{* *}$ & $-.46^{* *}$ & $.65^{* *}$ & - & & & \\
\hline 6. BNPS (Apoyo) & 3.78 & 0.68 & -.11 & $-.36^{* *}$ & $-.43^{* *}$ & $.69^{* *}$ & $.57^{* *}$ & - & & \\
\hline 7. Vulnerabilidad en línea & 2.78 & 0.99 & $.24^{* *}$ & $.32^{* *}$ & $.38^{* *}$ & $-.34^{* *}$ & $-.19^{* *}$ & -.30 & - & \\
\hline 8. Autoestima & 19.61 & 5.43 & $-.12^{*}$ & -.12 & $-.38^{* *}$ & $.63^{* *}$ & $.73^{* *}$ & $.60^{* *}$ & $-.17^{* *}$ & - \\
\hline
\end{tabular}

Estadísticos descriptivos y correlaciones entre variables. Notas: $\mathrm{n}=306: M=$ media; $D E=$ desviación estándar ${ }^{* *} p<0.01 .{ }^{*} p<0.05$. 
Tabla 2

\begin{tabular}{|l|c|c|c|c|c|c|c|c|}
\hline Variables & $\mathbf{1}$ & $\mathbf{2}$ & $\mathbf{3}$ & $\mathbf{4}$ & $\mathbf{5}$ & $\mathbf{6}$ & $\mathbf{7}$ & $\mathbf{8}$ \\
\hline 1. SMES & - & $.48^{* *}$ & $.36^{* *}$ & -.14 & -.16 & -.10 & $.25^{* *}$ & $-.19^{*}$ \\
\hline 2. BSMAS & $.57^{* *}$ & - & $.68^{* *}$ & $-.53^{* *}$ & $-.44^{* *}$ & $-.37^{* *}$ & $.42^{* *}$ & $-.35^{* *}$ \\
\hline 3. FOMO & $.50^{* *}$ & $.77^{* *}$ & - & $-.53^{* *}$ & $-.36^{* *}$ & $-.45^{* *}$ & $.39^{* *}$ & $-.36^{* *}$ \\
\hline 4. BNPS (Autonomía) & $-.18^{*}$ & $-.48^{* *}$ & $-.49^{* *}$ & - & $.62^{* *}$ & $.73^{* *}$ & $-.39^{* *}$ & $.65^{* *}$ \\
\hline 5. BNPS (Competencia) & -.15 & $-.46^{* *}$ & $-.58^{* *}$ & $.70^{* *}$ & - & $.59^{* *}$ & $-.16^{*}$ & $.74^{* *}$ \\
\hline 6. BNPS (Apoyo) & $-.16^{*}$ & $-.42^{* *}$ & $-.42^{* *}$ & $.65^{* *}$ & $.59^{* *}$ & - & $-.28^{* *}$ & $.65^{* *}$ \\
\hline 7. Vulnerabilidad en línea & $.24^{* *}$ & $.22^{* *}$ & $.38^{* *}$ & $-.29^{* *}$ & $-.22^{* *}$ & $-.34^{* *}$ & - & $-.20^{*}$ \\
\hline 8. Autoestima & -.04 & $-.36^{* *}$ & $-.40^{* *}$ & $.61^{* *}$ & $.71^{* *}$ & $.59^{* *}$ & -.15 & - \\
\hline
\end{tabular}

Correlaciones de hombres bajo la diagonal y mujeres sobre la diagonal. Notas: $n=306$; ${ }^{* *} p<0.01 .{ }^{*} p<0.05$.

En la Tabla 2 se observan las correlaciones de hombres bajo la diagonal y de mujeres sobre la diagonal. Mediante el $z$ test de Fisher se compararon los coeficientes de correlación de los dos grupos. Se nota como la relación entre las variables SMES e FOMO resulte estadísticamente mayor en los hombres ( $z=2.12, p=.017$ ); Lo mismo se observa considerando la relación entre BSMAS y FOMO ( $z=2.354, p=.009$ ), SMES y Autoestima ( $z=1.875$, $p=0.03)$ y entre el FOMO y la dimensión Competencia del BPNS $(z=3.515, p<0.001)$. Al contrario, la relación entre BSMAS y Vulnerabilidad en línea parece ser significativamente más fuerte en las mujeres $(z=-2.758, p=.003)$.

Las mujeres mostraron puntuaciones significativamente más altas en la escala BSMAS, $t(306)=2.07$, $p=.039 ; d=.24$ y en la dimensión Apoyo del BPNS, $t(306)=3.16, p=.002 ; d=.35$. En ambas variables, el tamaño del efecto resultó de media magnitud.

En las otras variables no se observaron diferencias estadísticamente significativas entre los grupos (Tabla 3).

Igualmente, se observaron puntaciones medias significativamente más altas de las mujeres en la frecuencia de uso de las redes sociales durante el horario universitario, $t(306)=3.92, p<.001)$, con

Tabla 3.

Comparación puntaciones media y desviación estándar de las variables según el sexo de los participantes.

\begin{tabular}{|l|c|c|}
\hline Variables & $\begin{array}{c}\text { Hombres (n=154) } \\
\mathbf{M}(\mathbf{D E})\end{array}$ & $\begin{array}{c}\text { Mujeres (n=152) } \\
\text { M (DE) }\end{array}$ \\
\hline SMES & $4.70(1.73)$ & $5.05(1.76)$ \\
\hline BSMAS & $2.11(0.82)$ & $2.30(0.78)$ \\
\hline FOMO & $2.22(0.81)$ & $2.25(0.79)$ \\
\hline BPNS (Autonomía) & $3.70(0.62)$ & $3.80(0.67)$ \\
\hline BPNS (Competencia) & $3.45(0.69)$ & $3.38(0.75)$ \\
\hline BPNS (Apoyo) & $3.66(0.67)$ & $3.90(0.66)$ \\
\hline Vulnerabilidad en línea & $2.79(0.96)$ & $2.77(1.01)$ \\
\hline Autoestima & $19.87(5.14)$ & $19.34(5.71)$ \\
\hline
\end{tabular}

Notas: $M=$ media; $D T$ = desviación estándar; 
Tabla 4.

Comparación puntaciones medias y desviación estándar en el tiempo de uso de aplicaciones y en la frecuencia de uso de redes sociales durante horario universitario según el sexo de los participantes.

\begin{tabular}{|l|c|c|}
\hline Variables & $\begin{array}{c}\text { Hombres (n=154) } \\
\mathbf{M}(\mathbf{D E})\end{array}$ & $\begin{array}{c}\text { Mujeres (n=152) } \\
\mathbf{M} \text { (DE) }\end{array}$ \\
\hline Instagram* & $3.11(0.96)$ & $3.53(0.97)$ \\
\hline WhatsApp* & $3.63(0.94)$ & $3.95(0.92)$ \\
\hline Facebook* & $2.12(0.90)$ & $2.14(0.86)$ \\
\hline Uso Redes sociales durante horario universitario** & $3.58(1.00)$ & $4.00(0.85)$ \\
\hline
\end{tabular}

Notas: $M=$ media; $D T$ = desviación estándar;

Tabla 5

Análisis de regresión

\begin{tabular}{|l|c|c|c|c|c|}
\hline \multirow{2}{*}{ Variables } & \multicolumn{5}{|c|}{ Modelo 1 } \\
\cline { 2 - 6 } & B $(\boldsymbol{\beta})$ & SE & $\mathbf{t}$ & p & IC 95\% \\
\hline (Constante) & 0.22 & 0.10 & 2.14 & 0.033 & {$[0.020 .42]$} \\
\hline FOMO & $0.62(0.61)$ & 0.04 & 14.94 & 0.000 & {$[0.540 .70]$} \\
\hline SMES & $0.12(0.27)$ & 0.02 & 6.60 & 0.000 & {$[0.090 .16]$} \\
\hline
\end{tabular}

Nota: Variable dependiente: BSMAS

un efecto de medio tamaño $(d=.43)$. Además, las mujeres mostraron puntaciones medias significativamente mayores en el tiempo de uso de Instagram respecto a los hombres, $t(306)=3.84, p<$ .001. El tamaño del efecto resultó de magnitud media $(d=.42)$. Lo mismo ocurre con WhatsApp, $t(306)=2.99, p=.003 ; d=.33$. No se encontraron diferencias de género estadísticamente significativas en el tiempo de uso de Facebook (Tabla 4).

Finalmente, el análisis de regresión lineal demostró como el FOMO ( $b=.61, p<.001)$ y la SMES $(b=.27, p<.001)$ resultaron predictores estadísticamente significativos de la variable BMSAS, es decir, del comportamiento potencialmente dependiente de redes sociales (Tabla 5). Las dos variables explican el 58.6\% de la varianza total de la variable criterio BSMAS $(R 2=.586, F(2,303)=214.38, p<.001)$.

\section{Discusión}

Casi el 71\% de los participantes declararon usar las redes sociales con mucha frecuencia durante el horario universitario. Este comportamiento parece ser normal, ya que aquellos que no lo implementan representan solo el $2 \%$ de la muestra. Específicamente, las mujeres revelaron una mayor frecuencia de uso de las redes sociales durante el horario universitario. El $96.4 \%$ de los participantes usa WhatsApp, el 95.1\% tiene al menos una cuenta de Instagram y el 69.9\% afirma usar Facebook. Las dos primeras aplicaciones muestran un uso diario promedio de aproximadamente 3 horas, mientras que la tercera parece ser la menos visitada en términos de horas diarias. Para la muestra de referencia, las mujeres muestran una mayor frecuencia en el uso de WhatsApp e Instagram.

La mayoría de los participantes afirmó que, en los últimos siete días revisaron sus sitios de redes sociales todos los días en los 15 minutos antes de acostarse y 15 minutos después de despertarse. En la práctica, para el $77.5 \%$ de los participantes, independientemente del género, las redes sociales son lo último que revisan antes de acostarse y para el 55.6\% es lo primero que revisan cuando se despiertan.

Las mujeres parecen mostrar una mayor tendencia a experimentar síntomas de adicción asociadas al uso de las redes sociales, como ya se 
encontró en Andreassen, Torsheim, Brunborg, \& Pallesen (2012) y en Monacis, De Palo, Griffiths \& Sinatra (2017) y a percibir un menor Satisfacción de la necesidad de Apoyo en la escala BPNS. Se encontraron resultados similares en la adaptación italiana del cuestionario BPNS (Szadejko, 2003). Es plausible que una de las razones detrás de estos resultados radique en sus disposiciones a percibir en mayor medida la necesidad de estrechar relaciones sociales significativas. Aspecto que puede satisfacerse parcialmente mediante la búsqueda de relaciones virtuales.

Finalmente, es interesante subrayar cómo el 72.9\% de los participantes declararon haber sido víctimas o haber visto a otros tropezar con comentarios críticos u ofensivos y el 70.6\% sufrir cotilleos y chismes perjudiciales para su propia reputación o la de otros. No hay diferencias de género para el área analizada.

El aumento de los niveles de Adicción a redes sociales también aumenta el miedo a ser excluido y a que otros estén viviendo experiencias gratificantes en propia ausencia.

Altos niveles de Adicción a redes sociales están relacionados con una baja percepción de satisfacción de las necesidades psicológicas básicas y con una baja autoestima. El vínculo entre los niveles de Adicción a redes sociales y la autoestima ya se había hecho evidente en varias investigaciones anteriores (Wilson, Fornasier \& White, 2010; Hong, Huang \& Chiu, 2014). Aún más fuerte es la correlación indirecta entre las tres dimensiones del BPNS y el FOMO.

A medida que aumentan las puntaciones del BSMAS, también aumenta el riesgo de experimentar situaciones potencialmente dañinas para la reputación, el bienestar psicológico y físico como resultado de las actividades realizadas en línea (Vulnerabilidad en línea). Lo mismo se aplica al FOMO, cuyos niveles están directamente relacionados con los de la Escala de la Vulnerabilidad en línea. Las puntuaciones altas en esta dimensión parecerían estar relacionadas con niveles más bajos de autoestima. Estas relaciones pueden ser más o menos fuertes según el género de los participantes.

Finalmente surgió que el FOMO y la intensidad del uso de las redes sociales durante la vida cotidiana (SMES) pueden considerarse predictores significativos de la Adicción a redes sociales, los cuales explican el 58.6\% de la varianza total.
La auto-selección de los encuestados y el número relativamente bajo de participantes no permite una generalización de los resultados. Además, es posible que los participantes hayan respondido siguiendo, más o menos conscientemente, el sesgo de prejuicio de la deseabilidad social.

Para compensar, en futuros estudios, podrá ser útil crear diarios en los que todos los participantes puedan proporcionar una descripción detallada del tiempo dedicado a las redes sociales, los métodos y las motivaciones, para aclarar las variables psicológicas subyacentes e identificar las modalidades de publicación.

Al ser un campo de investigación relativamente joven, en continua evolución dado el rápido cambio que acompaña al mundo digital, los resultados obtenidos también deberían replicarse en otros contextos culturales para adquirir más validez y confiabilidad. Mediante la aplicación de mejores proyectos metodológicos, incluidas muestras más representativas y heterogéneas, se podrán llenar los vacíos actuales en el conocimiento del fenómeno.

\section{Conclusiones}

Las redes sociales facilitan la difusión de información, favorecen las relaciones sociales y permiten una conexión entre las personas más allá de los límites del espacio-tiempo. Sin embargo, cualquier inclinación excesiva hacia una actividad puede resultar en una adicción, independientemente de si una sustancia química esté involucrada o no. Xu \& Tan (2012) sugieren que la transición del uso normal a problemático de las redes sociales ocurre cuando el individuo comienza a considerarlas como un mecanismo importante (o incluso exclusivo) para aliviar el estrés, la soledad o la depresión. El uso de las redes sociales puede proporcionar una serie de recompensas continuas (gratificaciones, autoeficacia, satisfacción de algunas necesidades) que empujan a las personas a involucrarse cada vez más en la misma actividad, hasta ignorar sus relaciones reales y encontrar diferentes problemas en la vida cotidiana.

De hecho, hay evidencias emergentes de que una minoría de usuarios de redes sociales exhiben síntomas adictivos como resultado del uso exce- 
sivo de diferentes aplicaciones (Griffiths, Kuss \& Demetrovics, 2014). El miedo a perderse experiencias y a ser excluido (FOMO) parece ser el principal factor de motivación que impulsa a las personas a encontrar un uso constante y excesivo de los sitios de redes sociales: el deseo de mantenerse siempre conectado con otros por miedo a perder experiencias gratificantes podría llevar a las personas a verificar compulsivamente sus sitios de redes sociales, incluso durante actividades que requieren un cierto grado de concentración, con el riesgo de encontrarse con problemas en el desempeño individual (Andreassen, Torsheim \& Pallesen, 2014), en la pérdida de empleos (Karaiskos, Tzavellas, Balta \& Paparrigopoulos 2010), calificaciones más bajas en la escuela y peor desempeño en el lugar de trabajo (Çam \& Isbulan, 2012; Koc \& Gulyagci, 2013).

La gran mayoría de los participantes dijo que usan sus sitios de redes sociales durante las principales actividades diarias: para muchos, representa la primera cosa de verificar después de despertarse y lo último que debe consultar antes de irse a dormir. Alrededor de un tercio de los participantes también verifican sus perfiles durante las comidas principales y durante las horas de universidad, comportamiento que podría atribuirse a la categoría Phubbing: por lo tanto, parece que la mayoría de los participantes se centre en su smartphone incluso cuando, según el contexto y las convenciones sociales, se contemplarían interacciones y/o atenciones entre los presentes. Podríamos hablar de una especie de retraimiento social impulsado por una preferencia por las relaciones virtuales a expensas de las reales.

El uso excesivo de las redes sociales y el deseo de no perderse ni siquiera una actualización o el intercambio de sus amigos virtuales, aumenta el riesgo de encontrar contenido inapropiado, $\mathrm{Ci}$ berbullying y problemas en el manejo de la privacidad. Puede ser más fácil acceder a contenido pornográfico, violento o racista, o visitar blogs que pueden empujar a los niños a cometer acciones criminales, inducirlos a mostrar coraje (tomar selfies en condiciones de riesgo), promover comportamientos anoréxicos y fomentar el consumo excesivo de alcohol o de drogas (Sgalla \& Giannini, 2018).

Los resultados obtenidos nos han permitido esbozar una imagen de los estilos de vida en línea y fuera de línea de los jóvenes en la era digital, pro- porcionando indicaciones útiles para proponer intervenciones específicas que permitan responder a las necesidades reales de las personas. Para las estrategias de prevención, jóvenes y adultos tienen responsabilidades mutuas: los primeros pueden capacitar a los segundos en el uso de las nuevas tecnologías, su lenguaje y sus posibilidades; Los segundos, a su vez, deben enseñar a los jóvenes a usarlos en su medida adecuada y ayudarlos a desarrollar la capacidad de comunicación cara a cara (Ramón-Cortés, 2010). El objetivo de un posible programa educativo que pueda dirigir correctamente a la población universitaria hacia un uso positivo de la tecnología debe comenzar por la aceptación de que el uso de tales aplicaciones representa un elemento integral de la cultura profesional y de ocio actual (Kuss \& Griffiths, 2011). En este sentido, Estrada (2004) resalta que es necesario desarrollar prácticas que empiezan por un análisis crítico de determinados aspectos culturales, de discursos aceptados, y por la reflexión acerca de los efectos de poder en los discursos en las instituciones. Es necesario identificar las distorsiones cognitivas, las emociones, las motivaciones y los factores de riesgo que subyacen a estos comportamientos desadaptativos. Para recuperar el control del comportamiento y prevenir cualquier acto inmoral se sugieren técnicas y métodos de Construcción del yo: mejor comprensión de diálogos, autoexpresión, autorregulación; Reflexión sociomoral: análisis crítico de la situación, construcción de conceptos, utilizo de dilemas morales; y la Convivencialidad: técnicas de role-playing; y desarrollo de habilidades sociales (Martí-Vilar, 2008). Podría ser útil, además limitar el uso de dispositivos y acordar las horas de uso de dispositivos electrónicos, promover pasatiempos, practicar deportes y participar en actividades de voluntariado (Echeburúa \& De Corral, 2010).

Es necesaria una comparación continua de modelos, teorías, estrategias de prevención y contraste para garantizar que la red sea un lugar seguro, que ayude y no obstaculice a sus usuarios (Sgalla \& Giannini, 2018). En esta área, la investigación tiene una tarea fundamental, que no debe ser hiper-patologizar el comportamiento diario, sino poner a disposición datos cada vez más confiables para facilitar apoyo a quienes lo necesiten. A partir de una mayor conciencia de tales mecanismos 
y de la frecuencia de tales comportamientos negativos, será posible trabajar sobre sus fortalezas y debilidades, tanto a nivel colectivo cuanto individual, con el objetivo de mejorar el ambiente de trabajo y de estudio fomentando valores de cooperación y de ética profesional.

Teniendo en cuenta que los conocimientos teóricos no son suficientes, la Universidad es la entidad más idónea para poder alcanzar estos objetivos. La respalda una fuerte implicación social e impacto sobre su entorno. La Responsabilidad Social Universitaria (RSU) se ha de concretizar garantizando un compromiso constante hacia la realización de cambios estructurales en sus cuatro pilares básicos. Es decir, su organización, el personal docente, el alumnado y el personal administrativo (Vallaeys, 2007).

Es importante que la universidad se encargue de enseñar al alumnado competencias éticas y de responsabilidad social incrementando la empatía de los estudiantes y su vinculación moral de estos con la sociedad (Martí-Vilar, Almerich, Cifuentes, Grimaldo, Martí, Merino, \& Puerta 2011).

\section{Referencias}

Andreassen, C. S. (2015). Online social network site addiction: A comprehensive review. Current Addiction Reports, 2(2), 175-184. https://doi.org/10.1007/s40429015-0056-9

Andreassen, C. S., Billieux, J., Griffiths, M. D., Kuss, D. J., Demetrovics, Z., Mazzoni, E., \& Pallesen, S. (2016). The relationship between addictive use of social media and video games and symptoms of psychiatric disorders: A large-scale cross-sectional study. Psychology of Addictive Behaviors, 30(2), 252. https://doi.org/10.1037/ adb0000160

Andreassen, C. S., Torsheim, T., \& Pallesen, S. (2014). Use of online social network sites for personal purposes at work: Does it impair self-reported performance? Comprehensive psychology, 3, 01-21. https://doi. org/10.2466/01.21.CP.3.18

Andreassen, C. S., Torsheim, T., Brunborg, G. S., \& Pallesen, S. (2012). Development of a Facebook addiction scale. Psychological reports, 110(2), 501-517. https://doi. org/10.2466/02.09.18.PRO.110.2.501-517

Binder, J. F., Howes, A., \& Smart, D. (2012). Harmony and ten- sion on social network sites: Side-effects of increasing online interconnectivity. Information, Communication \& Society, 15(9), 1279-1297. https://psycnet.apa. org/doi/10.1080/1369118X.2011.648949

Boyd, D. M., \& Ellison, N. B. (2007). Social network sites: Definition, history, and scholarship. Journal of computer-mediated Communication, 13(1), 210-230. https:// doi.org/10.1111/j.1083-6101.2007.00393.x

Buglass, S.L.; Binder, J.F.; Betts, L.R.; Underwood, J.D.M. (2016), Motivators of online vulnerability: The impact of social network site use and FOMO. Computers in $\mathrm{Hu}$ man Behavior, 66, 248-255. https://doi.org/10.1016/j. chb.2016.09.055

Burke, M., Marlow, C., \& Lento, T. (2010). Social network activity and social well-being. In Proceedings of the SIGCHI Conference on Human Factors in Computing Systems (pp. 1909-1912). https://doi. org/10.1145/1753326.1753613

Çam, E., \& Isbulan, O. (2012). A New Addiction for Teacher Candidates: Social Networks. Turkish Online Journal of Educational Technology-TOJET, 11(3), 14-19. Recuperado de https://www.researchgate.net/publication/267556427_A_new_addiction_for_teacher_candidates_Social_networks

Davidson, J., \& Martellozzo, E. (2013). Exploring young people's use of social networking sites and digital media in the internet safety context: A comparison of the UK and Bahrain. Information, Communication \& Society, 16(9), 1456-1476. https://doi.org/10.1080/1369118X.2012.701655

Deci, E. L., \& Ryan, R. M. (1985). Intrinsic motivation and self-determination in human behavior. New York: Plenum. https://doi.org/10.1007/978-1-4899-2271-7

Deci, E. L., \& Ryan, R. M. (2000). The" what" and" why" of goal pursuits: Human needs and the self-determination of behavior. Psychological inquiry, 11(4), 227-268. https:// doi.org/10.1207/S15327965PLI1104_01

Echeburúa, E., \& De Corral, P. (2010). Adicción a las nuevas tecnologías ya las redes sociales en jóvenes: un nuevo reto. Adicciones, 22(2), 91-96. https://doi.org/10.20882/ adicciones.196

Estrada, A. M. (2004). La psicología social en el concierto de la transdisciplinariedad. Retos latinoamericanos. Revista De Estudios Sociales, 18, 51-58. https://doi. org/10.7440/res18.2004.04

Goleman, D. (1995). Emotional intelligence. New York: Bantam Books.

Griffiths, M. D., Kuss, D. J., \& Demetrovics, Z. (2014). Social networking addiction: An overview of preliminary 
findings. Behavioral addictions (pp. 119-141). Academic Press. https://doi.org/10.1016/B978-0-12-4077249.00006-9

Hinduja, S., \& Patchin, J. W. (2010). Bullying, cyberbullying, and suicide. Archives of suicide research, 14(3), 206 221. https://doi.org/10.1080/13811118.2010.494133

Hong, F. Y., Huang, D. H., Lin, H. Y., \& Chiu, S. L. (2014). Analysis of the psychological traits, Facebook usage, and Facebook addiction model of Taiwanese university students. Telematics and Informatics, 31(4), 597-606. https://doi.org/10.1016/j.tele.2014.01.001

Joinson, A., McKenna, K.Y.A., Postmes, T. \& Reips, U.D. (2007). The Oxford Handbook of Internet Psychology. New York: Oxford University Press. https://doi.org/10.1093/ oxfordhb/9780199561803.001.0001

Jones, L. M., Mitchell, K. J., \& Finkelhor, D. (2013). Online harassment in context: Trends from three Youth Internet Safety Surveys (2000, 2005, 2010). Psychology of Violence, 3(1), 53. https://psycnet.apa.org/doi/10.1037/ a0030309

Karaiskos, D., Tzavellas, E., Balta, G., \& Paparrigopoulos, T. (2010). P02-232-Social network addiction: a new clinical disorder?. European Psychiatry, 25, 855. https:// doi.org/10.1016/S0924-9338(10)70846-4

Keipi, T., Oksanen, A., Hawdon, J., Näsi, M., \& Räsänen, P. (2015). Harm-advocating online content and subjective well-being: a cross-national study of new risks faced by youth. Journal of Risk Research, 1-16. https://doi.org/1 0.1080/13669877.2015.1100660

Kim, J., \& Lee, J. E. (2011). The Facebook paths to happiness: Effect of the number of Facebook friends and selfpresentation on subjective well-being. Cyberpsychology, Behavior, and Social Networking, 14, 359-364. https://doi.org/10.1089/cyber.2010.0374

Kim, J., \& Tussyadiah, I. P. (2013). Social networking and social support in tourism experience: The moderating role of online self-presentation strategies. Journal of Travel \& Tourism Marketing, 30(1-2), 78-92. https://doi.or $\mathrm{g} / 10.1080 / 10548408.2013 .751220$

Koc, M., \& Gulyagci, S. (2013). Facebook addiction among Turkish college students: The role of psychological health, demographic, and usage characteristics. Cyberpsychology, Behavior, and Social Networking, 16(4), 279-284. https://doi.org/10.1089/cyber.2012.0249

Krämer, C. N., \& Winter, S. (2008). Impression management 2.0: The relationship of self-esteem, extraversion, self-efficacy, and self-presentation within social networking sites. Journal of Media Psychology, 20, 106116. https://doi.org/10.1027/1864-1105.20.3.106

Kuss, D. J., \& Griffiths, M. D. (2011). Online social networking and addiction-a review of the psychological literature. International journal of environmental re search and public health, 8(9), 3528-3552. https://doi. org/10.3390/ijerph8093528

La Guardia, J. G., Ryan, R. M., Couchman, C. E., \& Deci, E. L. (2000). Basic Psychological Needs Scales. Journal of Personality and Social Psychology, 79, 367-384. https://doi.org/10.1037/0022-3514.79.3.367

Landoll, R. R., La Greca, A. M., Lai, B. S., Chan, S. F., \& Herge, W. M. (2015). Cyber victimization by peers: Prospective associations with adolescent social anxiety and depressive symptoms. Journal of adolescence, 42, 77-86. https://doi.org/10.1016/j.adolescence.2015.04.002

LeFebvre, L. E. (2018). Swiping me off my feet: Explicating relationship initiation on Tinder. Journal of Social and Personal Relationships, 35(9), 1205-1229. https://doi. org/10.1177\%2F0265407517706419

Lenhard, W., \& Lenhard, A. (2014). Hypothesis tests for comparing correlations. Bibergau: Psychometrica. Recuperado de https://www.psychometrica.de/correlation.html.

Li, X., \& Chen, W. (2014). Facebook or Renren? A comparative study of social networking site use and social capital among Chinese international students in the United States. Computers in Human Behavior, 35, 116-123. https://doi.org/10.1016/j.chb.2014.02.012

Livingstone, S., \& Smith, P. K. (2014). Annual research review: Harms experienced by child users of online and mobile technologies: The nature, prevalence and management of sexual and aggressive risks in the digital age. Journal of child psychology and psychiatry, 55(6), 635-654. https://doi.org/10.1111/jcpp.12197

Martí-Vilar, M. (2008). Las necesidades humanas desde la psicología moral. Papeles de relaciones ecosocialesy cambio global, 102, 89-101. Recuperado de https:// dialnet.unirioja.es/servlet/articulo?codigo=2698952.

Martí-Vilar, M., G. Almerich, J. Cifuentes, M. Grimaldo, Martí, J., Merino, C., \& Puerta, I.C. (2011). Responsabilidad Social Universitaria: Estudio iberoamericano sobre la influencia de la educación en la formación de profesionales responsables con la sociedad. Técnica Administrativa, 10(47), 3-10. https://doi.org/10.1016/S01200534(14)70019-6

Monacis, L., De Palo, V., Griffiths, M. D., \& Sinatra, M. (2017). Social networking addiction, attachment style, and validation of the Italian version of the Bergen Social Media Addiction Scale. Journal of Behavioral Addictions, 6(2), 178-186. https://doi. org/10.1556/2006.6.2017.023

Patchin, J. W., \& Hinduja, S. (2010). Changes in adolescent online social networking behaviors from 2006 to 2009. 
Computers in Human Behavior, 26(6), 1818-1821. https://psycnet.apa.org/doi/10.1016/j.chb.2010.07.009

Prezza, M., Trombaccia, F. R., e Armento, L. (1997). La scala dell'autostima di Rosenberg: Traduzione e validazione italiana. Bollettino di Psicologia Applicata, 223, 35-44. Recuperado de https://psycnet.apa.org/record/1998-01058-003.

Przybylski, A. K., Murayama, K., DeHaan, C. R., \& Gladwell, V. (2013). Motivational, emotional, and behavioral correlates of fear of missing out. Computers in Human Behavior, 29(4), 1841-1848. https://doi.org/10.1016/j. chb.2013.02.014

Przybylski, A. K., Weinstein, N., Ryan, R. M., \& Rigby, C. S. (2009). Having to versus wanting to play: Background and consequences of harmonious versus obsessive engagement in video games. CyberPsychology \& Behavior, 12(5), 485-492. https://doi.org/10.1089/ cpb.2009.0083

Ramón-Cortés, F. (2010). ¿Internet amenaza el contacto real? El país semanal, 1736, 24-25. Recuperado de https:// elpais.com/diario/2010/01/03/eps/1262503611_850215. html

Robins, R. W., Hendin, H. M., \& Trzesniewski, K. H. (2001). Measuring global self-esteem: Construct validation of a single-item measure and the Rosenberg Self-Esteem Scale. Personality and social psychology bulletin, 27(2), 151-161. https://doi.org/ 10.1177\%2F0146167201272002

Rosenberg, M. (1965). Society and the adolescent self-image. Princeton, NJ: Princeton University Press. http://doi. org/10.1126/science.148.3671.804

Sgalla, R., \& Giannini, A.M. (2018). La Violenza in un clic. Itinerari di prevenzione del cyberbullismo fra adolescenti. Firenze: Hogrefe

Staksrud, E., Ólafsson, K., \& Livingstone, S. (2013). Does the use of social networking sites increase children's risk of harm? Computers in Human Behavior, 29(1), 40-50. https://doi.org/10.1016/j.chb.2012.05.026

Sumter, S.R., Vandenbosch, L., Ligtenberg, L., 2017. Love me Tinder: untangling emerging adults' motivations for using the dating application Tinder. Telematics and Informatics. 34 (1), 67e78. https://doi.org/10.1016/j. tele.2016.04.009
Szadejko, K. (2003). Percezione di autonomia, competenza e relazionalità. Adattamento italiano del questionario basic psychological needs scale. Orientamenti pedagogici, 50(299), 853-872. Recuperado de https://dialnet.unirioja.es/servlet/articulo?codigo=3534145

UNESCO. (2014). Global citizenship education: Preparing learners for the challenges of the twenty-first century. Recuperado de http://unesdoc.unesco.org/images/0022/002277/227729E.pdf

Vallaeys, F. (2007). Responsabilidad social universitaria. Propuesta para una definición madura y eficiente. Programa para la formación en humanidades. Tecnológico de Monterrey. Recuperado de http://www. responsable.net/sites/default/files/responsabilidad_ social_universitaria_francois_vallaeys.pdf

Villacampa, J., Ingram, G. P., Martí-Vilar, M., \& Olivera-La Rosa, A. (2018). An investigation of Facebook users' implicit associations between Facebook, sexual and prosocial behavior. Heliyon, 4(9), e00811. https://doi.org/10.1016/j.heliyon.2018.e00811

Washington Post, 2013. “Cyberbullying charges weighed after suicide of Florida girl, 12". Recuperado de http://www. washingtonpost.com/national/cyberbullying-charges-35

Weiser, D.A., Niehuis, S., Flora, J., Punyanunt-Carter, N.M., Arias, V.S., Baird, R.H., 2017. Swiping right: sociosexuality, intentions to engage in infidelity, and infidelity experiences on Tinder. Examining Personality and Individual Differences in Cyberspace, Personality and Individual Differences, 133: 29-33. https://psycnet.apa. org/doi/10.1016/j.paid.2017.10.025

Wilson, K., Fornasier, S., \& White, K. M. (2010). Psychological predictors of young adults' use of social networking sites. Cyberpsychology, behavior, and social networking, 13(2), 173-177. https://psycnet.apa.org/ doi/10.1089/cyber.2009.0094

Xu, H., \& Tan, B. C. (2012). Why do I keep checking Facebook: Effects of message characteristics on the formation of social network services addiction. International Conference on Information Systems, ICIS 2012, 1, 812823. Recuperado de https://pdfs.semanticscholar.org/447a/7e658f27eab41279352ecac71b97f42abfc3.pdf

RIDU / Revista Digital de Investigación en Docencia Universitaria / e-ISSN: 2223-2516

๑ Los autores. Este artículo es publicado por la Revista Digital de Investigación en Docencia Universitaria del Área de Institutional Research and Effectiveness de la Dirección de Aseguramiento de la Calidad, Universidad Peruana de Ciencias Aplicadas. Este es un artículo de acceso abierto, distribuido bajo los términos de la LicenciaCreativeCommons Atribución-CompartirIgual 4.0 Internacional.( http://creativecommons.org/licenses/by-sa/4.0/), que permite el uso no comercial, distribución y reproducción en cualquier medio, siempre que la obra original sea debidamente citada. 